\title{
Laser micromarking technique in studying the negative gravitropism in pea stem
}

\author{
Akira Watanabe ${ }^{1, *}$, Ashiqur Rahman ${ }^{1}$, Kazuhiko Nishitani ${ }^{2}$, Rie Yamada ${ }^{3}$, \\ Mariko Takahashi ${ }^{3}$, Satoru Tsugawa ${ }^{4}$, Taku Demura ${ }^{4}$ \\ ${ }^{1}$ Institute of Multidisciplinary Research for Advanced Materials, Tohoku University, 2-1-1 Katahira, Aoba-ku, Sendai-shi, \\ Miyagi 980-8577, Japan; ${ }^{2}$ Department of Biological Sciences, Faculty of Science, Kanagawa University, 2946 Tsuchiya, \\ Hiratsuka-shi, Kanagawa 259-1293, Japan; ${ }^{3}$ Tohoku Electronic Industrial Co., Ltd., 2-14-1 Mukaiyama, Taihaku-ku, Sendai- \\ shi, Miyagi 982-0841, Japan; ${ }^{4}$ Division of Biological Science, Nara Institute of Science and Technology (NAIST), 8916-5 \\ Takayamacho, Ikoma-shi, Nara 630-0192, Japan \\ *E-mail: akira.watanabe.c6@tohoku.ac.jp Tel \& Fax: +81-22-217-5851
}

Received June 13, 2020; accepted September 23, 2020 (Edited by T. Kotake)

\begin{abstract}
A laser micromarking technique on plant epidermis was developed to study how a plant can reduce the stress in bending behavior by controlling the growth and morphogenesis. The negative gravitropism in a pea seedling (Pisum sativum L.) was discussed based on the time-dependent displacement of laser marking points which were formed by spatiallyselective laser ablation of the cuticle layer that covers the outer surface of a plant. The elongation of the stem in the horizontal direction was remarkable in the first half of the gravitropism. The elongation percentages of the stem length between lasermarking points at around upper surface, middle, and bottom surface were evaluated to be $2.57,4.87$, and $7.70 \%$, respectively. The characteristic feature of the stem bending in gravitropism is the elongation even at the upper surface region, that is, inside of the bending. This is a different feature from cantilever beams for structural materials like metals and polymers, where the compression of the upper surface and elongation of the bottom surface are caused by bending. Another laser micromarking technique was developed to improve the resolution of a dot-matrix pattern by fluorescent material transfer to a plant through a masking film with a micro-hole matrix pattern. Similar time-dependent displacement behavior was observed for a fluorescent dot-marked stem showing a feedback control loop in the mechanical optimization. These results suggested that plants solve the problem of the stress in stem bending through growth. The laser micromarking is an effective method for studying the mechanical optimization in plants.
\end{abstract}

Key words: gravitropism, laser micromarking, mechanical optimization in plants, stem bending.

Plants optimize the size, shape and organs under external loads such as wind, rain, temperature, light, gravity, neighboring structures and internal loads such as plant's own weight, turgor pressure in cells, and so on. Beam models have been applied for the bending of a plant stem in the field of mechanobiology (Moulia et al. 2015). A beam is a structural element of building to withstand a load. A beam of any elastic material shows a change in shape under external loads. When a simply supported structural beam is loaded downwards at the middle, the upper surface of the bending beam is in compression and the bottom surface is in tension. Figure 1 is the image of static stress simulation for a cantilever beam based on finite element analysis (FEA) by a computer aided design software (Fusion 360), where a low-density polyethylene (LDPE) rod with the size of $40 \mathrm{~mm}$ long and $2 \mathrm{~mm}$ diameter was loaded with a concentrated load of $0.1 \mathrm{~N}$ upwards at the right-hand edge. The upward bending of the LDPE rod was shown with the stress distribution. The maximum compression and tension stresses indicated by red color are distributed around the upper and bottom surfaces at the left-hand edge, respectively. The blue color is assigned to the unstressed region and distributed at the center of the beam, which is called the neutral axis (NA) of a beam. Such a stress distribution caused the compression of the upper surface and elongation of the bottom surface. On the other hand, NA region showed no change in the length. Such distortions are characteristic of a bending beam consisting of structural materials. An overload distortion causes the risk of breakages. In this paper, we report the mechanical optimization process in plants studied by using laser micromarking technique on a plant stem. The issue in this study is how a plant can reduce the stress in bending by controlling the growth and morphogenesis. As a bending behavior of a plant, the gravitropism in a pea seedling (Pisum sativum L.) was studied by using laser micromarking technique. Plant tropisms are the 


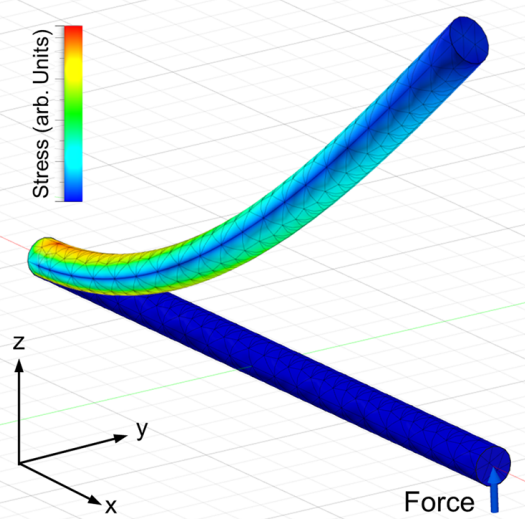

Figure 1. Static stress simulation image for a cantilever beam of a low density polyethylene rod with the size of $40 \mathrm{~mm}$ long and $2 \mathrm{~mm}$ diameter. Force: $0.1 \mathrm{~N}$ upwards at the right-hand edge.

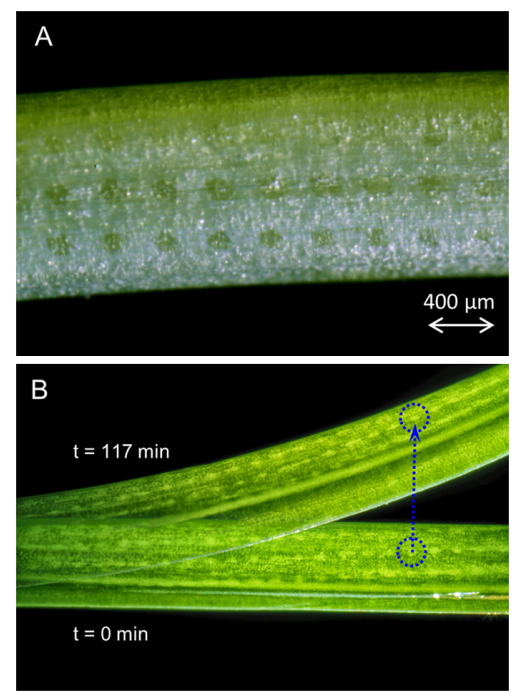

Figure 2. Macro-photographic images of a dot-matrix pattern directly drawn on a pea seedling (Pisum sativum L.) stem with spacing distance of $400 \mu \mathrm{m}$ (A) and a laser-marked pea seedling stem before and after gravitropism (B).

mechanisms by which plants optimize the shape to environmental changes. Gravitropism is important in plants as it directs stem growth in the opposite direction to gravity (negative gravitropism) (Morita 2010; Sack 1991). The displacement of a plant stem in gravitropism was studied by measuring the position changes for small dot matrix on a plant stem.

Laser micromarking on a plant stem was conducted by using a nanosecond pulse laser $(355 \mathrm{~nm}, 30 \mathrm{kHz}, 3 \mathrm{~W}$, FWHM 13 ns, INNGU LASER Pulse 355-3W). The laser beam was scanned by a galvano-scanner (MASTER LASER) with an $\mathrm{f}-\theta$ lens $(\mathrm{F}=100 \mathrm{~mm})$. Figure $2 \mathrm{~A}$ shows the macro-photographic image of a dot-matrix pattern directly drawn on a pea seedling stem, where the spacing distance among dot patterns was $400 \mu \mathrm{m}$. The formation of dot pattern can be attributed to the laser-ablation of the cuticle layer that covers the outer surface of a plant.
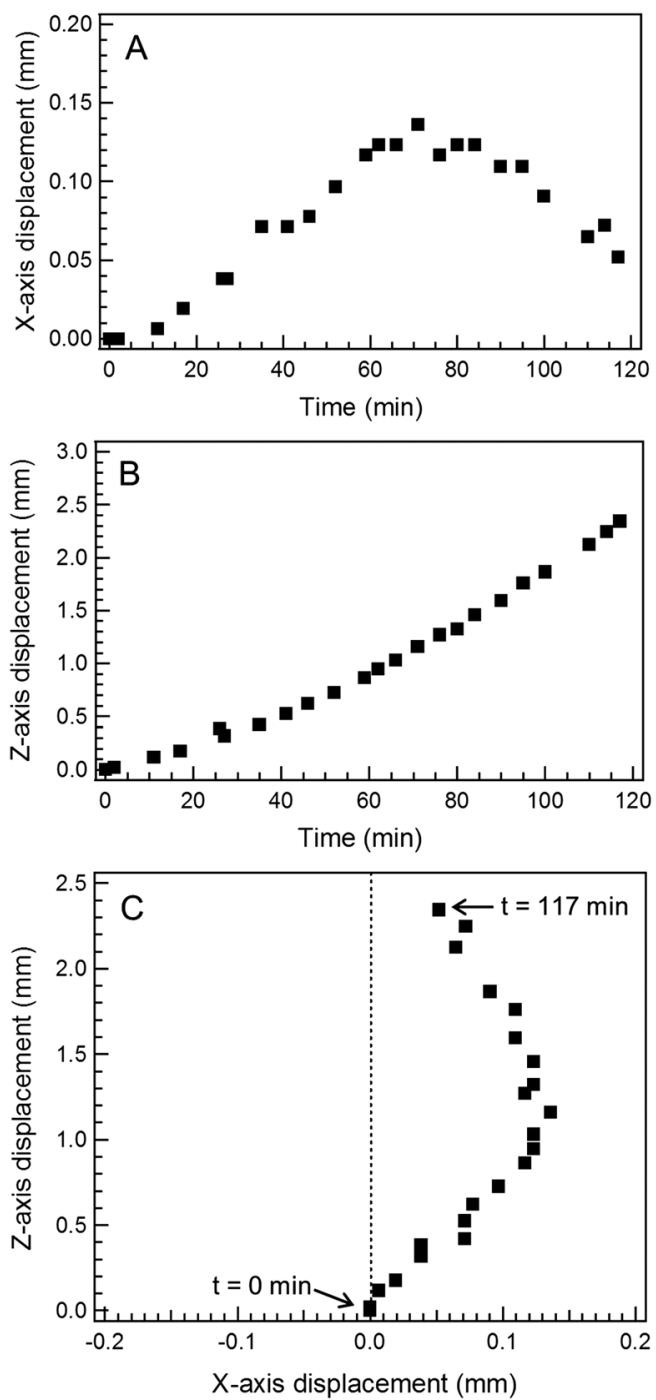

Figure 3. Time-dependent displacements of a laser-marked point during gravitropism. (A) X-axis displacement, (B) Z-axis displacement, and $(\mathrm{C}) \mathrm{X}-\mathrm{Z}$ plane displacement.

In this type of the laser micromarking, the dot size was several tens micrometer because of the heat formation at the laser-irradiated point although the spot size of the focused laser beam was several micrometer.

The displacements of laser marking dots were recorded by a digital camera (61 megapixels, Sony $\alpha 7 \mathrm{R} \mathrm{IV)}$ equipped with a macro lens (Zhongyi Mitakon $20 \mathrm{~mm}$ F2 4.5x) partially irradiating a white LED light on a pea seedling stem through an optical fiber, where a cut pea seedling stem was set in a sample tube bottle with a hydroponic sponge and water. The upward bending of a pea seedling stem in this experiment can be attributed to the gravitropism mainly because the white LED light was partially irradiated on a narrow region of the stem from diagonally below. In addition, gravitropism is the dominant tropic response in the early stage of bending compared with phototropism (Franssen 1980; Fukaki et al. 1996; Hart and Macdonald 1981). The macro- 


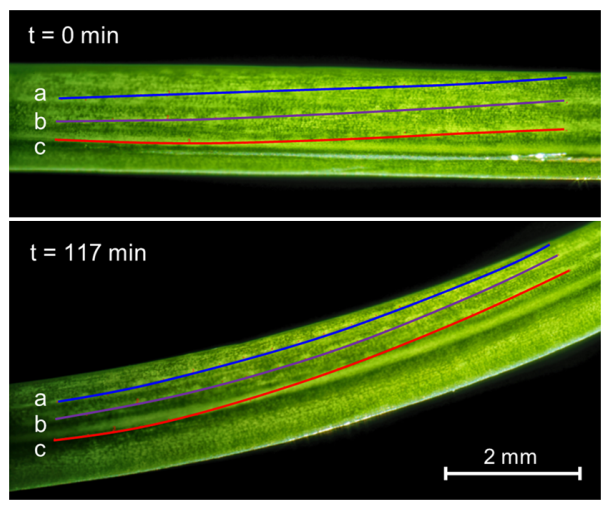

Figure 4. Length changes between right and left hand laser-marking points before and after gravitropism.

Table 1. Elongation percentages at around upper surface $a$, middle $b$, and bottom surface $\mathrm{c}$ of a stem in gravitropism.

\begin{tabular}{cccc}
\hline Position & $\begin{array}{c}\text { Length before } \\
\text { gravitropism }(\mathrm{mm})\end{array}$ & $\begin{array}{c}\text { Length after } \\
\text { gravitropism }(\mathrm{mm})\end{array}$ & $\begin{array}{c}\text { Elongation } \\
(\%)\end{array}$ \\
\hline a & 7.40 & 7.59 & 2.57 \\
$\mathrm{~b}$ & 7.39 & 7.75 & 4.87 \\
$\mathrm{c}$ & 7.40 & 7.97 & 7.70 \\
\hline
\end{tabular}

photographic images before and after gravitropism are shown in Figure 2B. The time-dependent displacement of a laser-making point indicated by a blue broken-line circle during gravitropism was analyzed and plotted in Figure 3. The $\mathrm{X}$-axis displacement in the horizontal direction of stem elongation showed the increase at the early stage of the gravitropism and then decreased after 70 min (Figure 3A). The Z-axis displacement in the direction of negative gravitropism showed a gradual increase of slope in the upward curve (Figure 3B). The trajectory of the laser marking point on the plant stem in $\mathrm{X}-\mathrm{Z}$ plane is shown in Figure 3C. The elongation of the stem to the horizontal direction was remarkable in the first half of the gravitropism. The bending of the stem caused the decrease of X-displacement in the latter half of the gravitropism.

The elongation percentage of the stem length between right and left hand laser-marking points was evaluated as shown in Figure 4, where the elongation percentages for different positions of the stem were compared at around upper surface $a$, middle $b$, and bottom surface $c$ of the stem in gravitropism. The lengths before and after gravitropism and the elongation percentages are summarized in Table 1 . The elongation percentages at around upper surface $a$, middle $b$, and bottom surface c are $2.57,4.87$, and $7.70 \%$, respectively. The growth in elongation even at the upper surface region, that is, inside of the bending is characteristic of a stem bending in gravitropism. This is a different feature from a cantilever beam as shown in Figure 1, where the compression of the upper surface and elongation of the bottom surface were caused by bending.
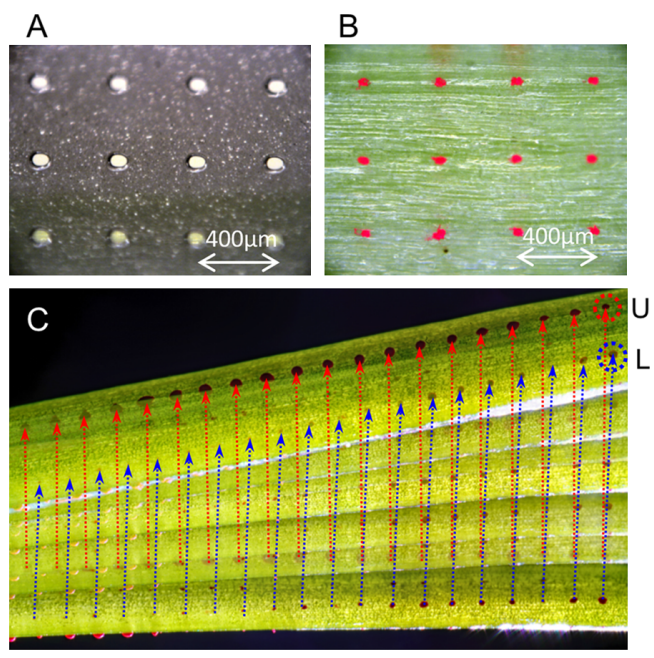

Figure 5. Formation of a fluorescent dot-matrix pattern on a pea seedling stem. (A) micro-hole matrix pattern prepared on a masking tape covering a pea seedling surface, (B) fluorescent dot-matrix pattern on a pea seedling surface prepared by coating a fluorescent paint through the micro-hole matrix pattern, (C) macro-photographic images of a fluorescent dot-marked stem during gravitropism. Red and blue arrows shows the displacements of fluorescent dots marked at the upper and bottom surfaces, respectively.

Another laser micromarking technique was developed to improve the resolution of a dot-matrix pattern by fluorescent material transfer through a masking film with a laser-written hole-matrix pattern. A thin black masking tape (Teraoka) was pasted on a pea seedling stem, which further reduced the damage of a plant during laser marking because the black masking tape has a high absorption efficiency for the $355 \mathrm{~nm}$ laser beam. A fluorescent dot-matrix pattern was formed on a pea seedling stem by coating a fluerescent paint (TURNER Lumi. Red) on the mask film and then peeling off it from the pea seedling stem. Figure 5A, B show a micro-hole matrix pattern prepared on a masking tape covering a pea seedling surface and a fluorescent dot-matrix pattern on a pea seedling surface. The advantages of the fluerescent marking technique compared to the direct marking on the cuticle layer are the smaller marking size and the higher visibility. When the direct laser marking on a plant in a laboratory is difficult, for example, in the cases of the marking on outdoor plants or large size plants, the fluerescent marking technique can be applied them by using a previously prepared masking film on a mold release film and pasting it on a plant. It is expected that such a marking technique can be applicable to various kinds of plants.

Figure $5 \mathrm{C}$ shows the macro-photographic images of a fluorescent-dot marked stem during gravitropism. The displacement of fluorescent dots marked at the upper and bottom surfaces are indicated by red and blue arrows, respectively. The red arrows for the upper surface dotmarks are almost in a perpendicular direction. The timedependent displacements for right-hand dots indicated 

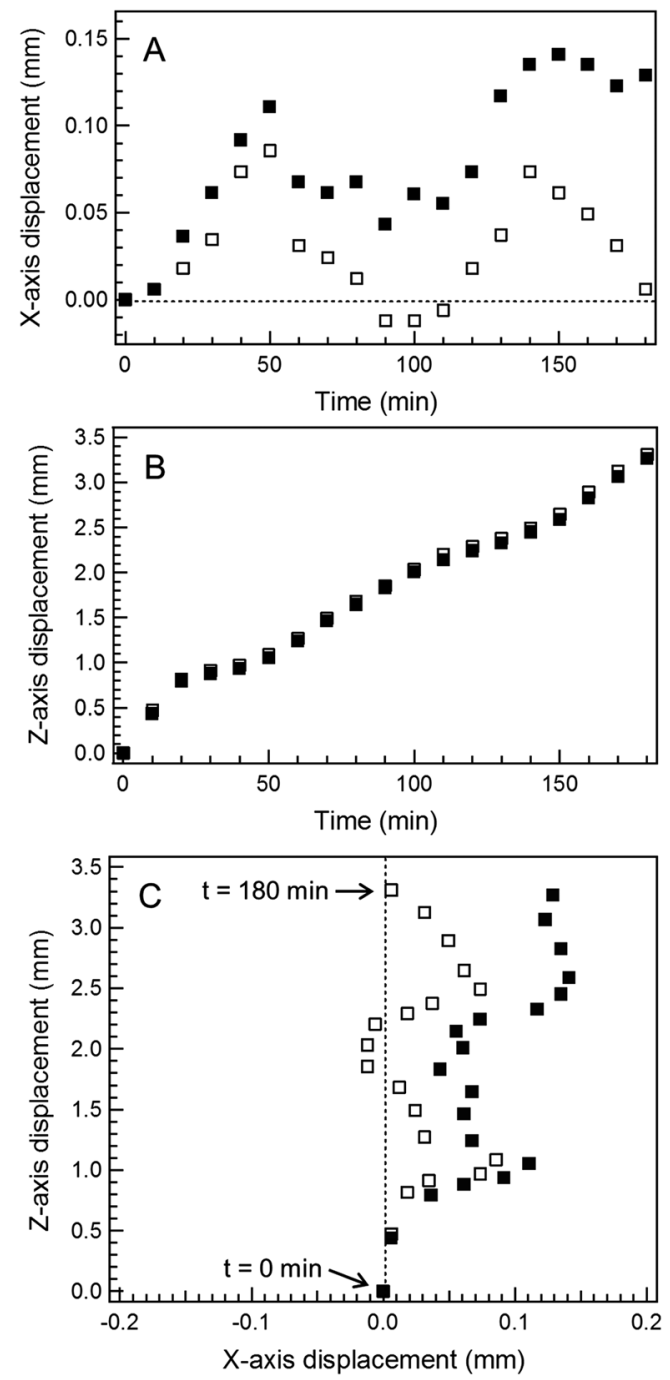

Figure 6. Time-dependent displacements of fluorescent-dots marked at the upper (open square) and bottom (filled square) surfaces. (A) $\mathrm{X}$-axis displacement, (B) Z-axis displacement, and (C) $\mathrm{X}-\mathrm{Z}$ plane displacement.

by $\mathrm{U}$ and $\mathrm{L}$ (Figure $5 \mathrm{C}$ ) are plotted in Figure 6 . The $\mathrm{X}$-axis displacement in the horizontal direction of the stem elongation prior to the stem bending as shown in Figure $3 \mathrm{~A}$ was observed again. The repearting cycle of such a displacement behavior was observed as shown in Figure 6A, C, which seems to be a feedback control loop in the mechanical optimization in plants. Plants solve the problem of the stress in stem bending through growth. Plant hormones, like auxins, are thought to help regulate the differential growth of a plant organ optimizing morphogenesis of plants (Kato et al. 2002; Okamoto et al. 2015; Taniguchi et al. 2017; Yoshihara et al. 2013). Such a behavior is quite different from the properties of structural materials like metals, polymers, and so on. In addition, the differences in the shape of a plant stem such as thickness, straightness, epicotyl length, hypocotyl length, position of leaf and so on change the mechanical balance of the stem in gravitropism and growth, which cause the complex dynamic behavior as shown in the difference between Figure 3A and Figure 6A. In the case of a plant which has non-uniform structute, the homogeneous bending of a plastic rod as shown in Figure 1 may be unsuitable, and so the complex dynamic behavior like a feedback control loop in the mechanical optimization may be necessaty.

In this study, we successfully developed a laser micromarking technique on plant epidermis which can be applied to the dynamic behavior study of a plant. The time-dependent displacement behavior of a lasermarked pea seedling stem in gravitropism was studied. In the negative gravitropism, the elongation of the stem was observed both at the upper and bottom surfaces. Plants solve the problem of the stress in stem bending by optimizing the stem length through growth. The laser micromarking is expected to be an effective method for studying the mechanical optimization in plants.

\section{Acknowledgements}

This work was supported by MEXT KAKENHI Grant-in-Aid for Scientific Research on Innovative Areas "Plant-Structure Optimization Strategy" Grant Numbers JP19H05356.

\section{References}

Franssen JM (1980) Phototropism in seedlings of sunflower Helianthus annuus L. Meded Landbouwhogeschool Wageningen 80: $1-83$

Fukaki H, Fujisawa H, Tasaka M (1996) Gravitropic response of inflorescence stems in Arabidopsis thaliana. Plant Physiol 110: 933-943

Hart JW, Macdonald IR (1981) Phototropism and geotropism in hypocotyls of cress (Lepidium sativum L.). Plant Cell Environ 4: 197-201

Kato T, Morita MT, Fukaki H, Yamauchi Y, Uehara M, Niihama M, Tasaka M (2002) SGR2, a phospholipase-like protein, and ZIG/SGR4, a SNARE, are involved in the shoot gravitropism of arabidopsis. Plant Cell 14: 33-46

Morita MT (2010) Directional gravity sensing in gravitropism. Annu Rev Plant Biol 61: 705-720

Moulia B, Coutand C, Julien JL (2015) Mechanosensitive control of plant growth: Bearing the load, sensing, transducing, and responding. Front Plant Sci 6: 1-20

Okamoto K, Ueda H, Shimada T, Tamura K, Kato T, Tasaka M, Morita MT, Hara-Nishimura I (2015) Regulation of organ straightening and plant posture by an actin-myosin XI cytoskeleton. Nat Plants 1: 15031

Sack FD (1991) Plant gravity sensing. Int Rev Cytol 127: 193-252

Taniguchi M, Furutani M, Nishimura T, Nakamura M, Fushita T, Iijima K, Baba K, Tanaka H, Toyota M, Tasaka M, et al. (2017) The arabidopsis LAZY1 family plays a key role in gravity signaling within statocytes and in branch angle control of roots and shoots. Plant Cell 29: 1984-1999

Yoshihara T, Spalding EP, Iino M (2013) tLAZY 1 is a signaling component required for gravitropism of the arabidopsis thaliana inflorescence. Plant J 74: 267-279 